\title{
Editorial
}

\section{The neglected tropical diseases in Sri Lanka}

\author{
De Silva $\mathrm{N}^{1 *}$ \\ ${ }^{1}$ Senior Professor of Parasitology, Faculty of Medicine, University of Kelaniya, Ragama, Sri Lanka
}

\begin{abstract}
Keywords: Neglected tropical diseases; Sri Lanka
Copyright: (C) 2014 De Silva N. This is an open access article distributed under the Creative Commons Attribution License, which permits unrestricted use, distribution, and reproduction in any medium, provided the original work is properly cited.
\end{abstract}

*Correspondence: nrdesilva@gmail.com

Cite this article as: De Silva N. The neglected tropical diseases in Sri Lanka. Anuradhapura Medical Journal 2014;8(2):30-33.

DOI: http://dx.doi.org/10.4038/amj.v8i2.7524

The term 'tropical diseases' was probably invented in the 19th century, when colonialism was widespread. It encompasses the diseases, mostly infectious, that the European invaders encountered for the first time in the tropics and subtropics. The term 'Neglected Tropical Diseases' (NTDs) is of rather more recent origin, having being coined by the World Health Organization (WHO) about ten years ago. The NTDs are a very diverse group with the commonality that all of them are strongly associated with poverty and social exclusion, and are often neglected in public health interventions. They consist of 17 communicable diseases now slated for global eradication, elimination or control by WHO (1). They include diseases caused by viruses (dengue, rabies), bacteria (Buruli ulcer, leprosy, trachoma, yaws), protozoa (Chagas disease, sleeping sickness, leishmaniasis) and helminths (taeniasis / cysticercosis, dracunculiasis, echinococcosis, food borne trematodiases, lymphatic filariasis, onchocerciasis, schistosomiasis and the soil-transmitted helminthiases). Many of these pathogens have very complex life cycles, population dynamics, infection processes and epidemiologies, all of which have contributed to their persistence.

In January 2012, WHO published its strategic plan for combating NTDs (2).The goals set out in this NTD Roadmap are ambitious. They include eradication of dracunculiasis by 2015 and yaws by 2020; the global elimination of blinding trachoma, leprosy, human African trypanosomiasis and lymphatic filariasis by 2020; and regional elimination of the other diseases by 2015 or 2020 . In this context, eradication is defined as the permanent reduction to zero of the worldwide incidence of infection caused by a specific pathogen, with no risk of reintroduction; while elimination (or interruption of transmission) is defined as the reduction to zero of the incidence of infection caused by a specific pathogen in a defined geographical area (3).

In May 2013, the Roadmap was endorsed by all members of the WHO at the 66th World Health Assembly (WHA
Resolution 66.12). Thus Sri Lanka has now committed itself to attaining the goals set out in the 2020 Roadmap on NTDs. Are we likely to meet these goals?

Sri Lanka is very fortunate in that unlike many other developing countries, we have to combat only six of the 17 NTDs: dengue, rabies, leprosy, leishmaniasis, lymphatic filariasis and the soil-transmitted helminthiases. Let us review the current status of each of these, especially with regard to progress towards achieving the goals of the WHO Roadmap on NTDs.

Dengue is the most problematic of the NTDs at present, being endemic in much of the country. The objectives of the NTD Roadmap are to reduce mortality from dengue by at least $50 \%$ by 2020 and reduce morbidity by at least $25 \%$, using 2010 as the baseline (2). According to national surveillance data released by the Epidemiology Unit of the Ministry of Health, there were 34,105 suspected or confirmed cases of DF / DHF during 2010, with a case fatality rate of $0.72 \%$. After declining to 28,140 cases and a case fatality rate of $0.63 \%$ in 2011 , the incidence shot up to 44,461 cases in 2012 , but the case fatality rate declined further, to $0.4 \%$ (4). The number of cases declined again to 31,975 in 2013 , with a further reduction of the case fatality rate, to $0.27 \%(5)$.

These figures suggest that Sri Lanka is well on its way to reaching the target relating to mortality reduction, but that morbidity reduction (as measured in terms of the incidence rate) is going to be much more difficult, particularly because an effective, commercially available dengue vaccine appears to be some years away. Without a vaccine, case reduction requires much greater efforts in sustainable vector control, along with integrated surveillance and outbreak response, coordinated by the National Dengue Control Programme.

Unlike dengue, rabies is a vaccine-preventable disease. The targets set out in the NTD Roadmap are to halve the number of human deaths due to rabies by 2016 , and to eliminate human rabies transmitted by dogs by 2020 in the WHO South East Asian Region (2). Sri Lanka has set itself 
a more ambitious target, to eliminate human rabies from the country by 2016 (4). The Public Health Veterinary Services (PHVS) is primarily responsible for preventing human and animal rabies. It has a well-articulated control strategy, which mainly focuses on mass vaccination of dogs, animal birth control and public awareness programmes. In the period since 2010 , about one million dogs have been vaccinated, and 150,000 - 200,000 dogs have been sterilized each year. This has resulted in a steady decline in the annual number of human rabies deaths, which have been recorded as 67, 52, 41, 38 and 28 during the period $2009-2013(4,5)$. These figures look promising, and Sri Lanka has the advantage of being an island, but in elimination programmes, the last mile is often the longest. If Sri Lanka is to meet its own target of eliminating human rabies deaths by 2016, the Ministry of Health and the public must support the PHVS in a strong, sustained effort to meet this goal.

Sri Lanka has been cited as implementing one of the most successful leprosy control programmes in public health history (6). Following a powerful social mobilization campaign, national prevalence dropped rapidly from nearly 8 per 10,000 population in 1990 , to below 1 per 10,000 population in 1995, thus meeting the WHO definition for elimination as a public health problem (6). Leprosy services were integrated into the general health services in 2001, and ownership for leprosy was transferred to the Provincial and District health services (4). However, the number of new cases has stubbornly remained at around 2000 cases a year for well over a decade, and the elimination target has yet to be achieved at sub-national level in districts such as Polonnaruwa (7).

In addition to the target of global elimination of leprosy as a public health problem by 2020 , the NTD Roadmap also targets a reduction of the global rate of new cases with grade- 2 disabilities per 1 million population, by at least $35 \%$ by the end of 2015 , compared with the baseline of 2010 (2). Sri Lanka's Anti-Leprosy Campaign has also set targets that focus on the early detection and management of cases: in 2010, 8\% of new cases had grade-2 disabilities; this is to be reduced by half, to $4 \%$ by the end of 2015 (4). Other targets include increasing the proportion of cases that are diagnosed within 6 months of onset of symptoms from $44 \%$ to $75 \%$; and improvement of treatment completion rates to $90 \%$ by the end of 2015 . So with leprosy too, the goal of elimination appears tantalizingly within reach, but a strong, sustained thrust will be required to achieve it by 2020 .

Leishmaniasis is a relatively new disease in Sri Lanka, the first case of indigenous cutaneous leishmaniasis (CL) being reported only in 1992 (8). As the war in the North and East intensified in the subsequent period, increasing numbers of cases were detected, and it was made a notifiable disease in 2008 (9). Since then, 674 cases were recorded in 2009, 428 in 2010, 930 in 2011, 1216 in 2012, followed by a very sharp decline to 131 cases in 2013 (5,10-12). A study on leishmaniasis in the Polonnaruwa District (which has the highest incidence) during the period 2008 - 2011, which combined data from dermatology clinics and the public health surveillance system, found that $43.4 \%$ of the patients were not reported to the surveillance system (13). This suggests that our current incidence records are likely to be underestimates, and the marked fall in the number of cases in 2013 may be simply due to non-notification. Although the vast majority of cases of leishmaniasis in Sri Lanka are of the cutaneous type, it has been clearly established that they are caused by Leishmania donovani (which causes the much deadlier visceral leishmaniasis (VL) in the Indian sub-continent), a few cases with visceral and muco-cutaneous manifestations have also been reported (9).

The targets for eliminating the leishmaniases in Asia, as set out in the NTD Roadmap, are to reduce the incidence of $\mathrm{VL}$ to $<1$ case / 10,000 population per year at district and sub-district levels in the South East Asia Region; and to detect and manage $85 \%$ of CL cases in all endemic countries by 2020 (2). Since Sri Lanka really doesn't have a problem with VL, that leaves only the latter target of detecting and managing $85 \%$ of CL cases. However, there is no separate entity within the Ministry of Health at present, with remit to monitor leishmaniasis or implement control measures. Indeed, there has been no definitive incrimination of the vector or the reservoir host(s) to date. In this vacuum, there is a danger that leishmaniasis may become much more of a problem than it is at present (9).

Sri Lanka has had a long-standing programme dedicated to the control of lymphatic filariasis (LF): the Anti-Filariasis Campaign (AFC) was established in 1947 (14). In 2002, Sri Lanka became one of the earliest countries to join the Global Programme for Elimination of Lymphatic Filariasis, which advocated for interruption of transmission through Mass Drug Administration (MDA) to endemic populations for at least five consecutive years. Sri Lanka successfully completed five rounds of MDA with diethylcarbamazine citrate and albendazole in 2006, covering more than $80 \%$ of the population residing in eight endemic districts (4). Transmission Assessment Surveys for post-MDA surveillance were carried out in 2008, 2009 and 2010 confirming that the microfilaraemia rate had dropped to well below 1\% (the WHO elimination target). In 2011, Sri Lanka qualified for commencement of the WHO verification process for certification of elimination (14). At present, the country dossier is being compiled by the AFC in readiness to submit it to the WHO later this year (Director, AFC, personal communication).

The soil-transmitted helminthiases (STH) have also been recognized as a problem for many years in Sri Lanka, but unlike all of the other NTDs, they are neither notifiable, nor is there a specific entity to monitor their control. A national survey of primary school children found the overall combined prevalence to be $6.9 \%$ in 2003 (15), but a subsequent survey of the plantation sector found $29.0 \%$ to have one or more STH infection, and $11.6 \%$ of the children had infections of moderate-heavy intensity (16). The plantation sector is a particularly high risk community because of the widespread poverty and lack of sanitation. A very recent survey in Gampaha district, which combined a LF Transmission Assessment Survey with STH, found 
that less than $3 \%$ of primary school children had STH infections (17). It is likely that the use of albendazole for the LF elimination programme during the period 2002 2006 drove down prevalence of STH in LF-endemic districts like Gampaha.

The NTD Roadmap targets for STH control are cognizant of the fact that although the biology of the STHs makes interruption of transmission extremely difficult, regular mass de-worming with anthelmintics is a very safe and cost-effective means of reducing morbidity. Hence the WHO target for 2015 is that $50 \%$ of pre-school and schoolaged children in need of treatment should be regularly treated in all countries, this figure reaching $75 \%$ by 2020 (2). The goal is to eliminate STH as a public health problem, such that less than $1 \%$ of the at-risk population has infection of moderate or high intensity (18).

In 2012, the Ministry of Health issued a new directive with guidelines on de-worming children and pregnant women in the community setting (19). These guidelines, which were developed by the Family Health Bureau, are to be implemented over the period 2013 - 2016. They categorize the country into high risk (Uva, Sabaraguwa and Central) and moderate risk provinces (all others), and recommend twice yearly and once yearly de-worming respectively, for children in these two areas. However, we have no national strategic plan that identifies explicit targets, milestones or performance indicators. This is an unfortunate situation, because given the current prevalence rates, it is very likely that Sri Lanka can eliminate STH as a public health problem in the near future (20)

Globally, there has been increasing recognition of the role played by NTDs as drivers and indicators of poverty. There have been widespread calls to include NTDs as subgoals in the post-2015 Sustainable Development Goals that will succeed the UN's Millenium Development Goals (21, 22). If this were to happen, it would be a goal that is well within Sri Lanka's reach. Achieving it will require sustained focus and commitment on the part of the Ministry of Health and all other stakeholders, but it will serve to reinforce Sri Lanka's image as a country with a health system that works for the poor and vulnerable as much as for the rich.

\section{References}

1. Working to overcome the global impact of neglected tropical diseases: first WHO report on neglected tropical diseases. Geneva, World Health Organization, 2010 (WHO/HTM/NTD/2010.1)

2. Accelerating work to overcome the global impact of neglected tropical diseases: a roadmap for implementation. Geneva, World Health Organization, 2012 (WHO/HTM/NTD/2012.1)

3. Sustaining the drive to overcome the global impact of neglected tropical diseases: second WHO report on neglected tropical diseases. Geneva, World Health Organization, 2013 (WHO/HTM/NTD/2013.1)

4. Performance and progress report 2012 - 2013. Colombo, Ministry of Health, 2013. (accessed at http://www.health.gov.lk/en/publication/P-PReport2012.pdf/PerformanceReport2012-E.pdf on 5 May 2014).

5. Gunasekera M. Flashback 2013. Weekly Epidemiological Report Sri Lanka 2014; 41 (1) 1 - 2.

6. Leprosy: learning from success. Geneva, World Health Organization, 2001 (WHO/CDS/CPE/CEE/2001.20)

7. Wijesinghe TS \& Wijesinghe PR. Integration of leprosy services into the general health service in Sri Lanka: overcoming challenges to implementation in a remote district. WHO South East Asia Journal of Public Health 2013; 2 (1) $63-68$.

8. Athukorale DN, Seneviratne JK, Ihalamulla RL, Premaratne UN. Locally acquired cutaneous leishmaniasis in Sri Lanka. Journal of Tropical Medicine\& Hygiene 1992; 95:432-433.

9. Karunaweera ND. Leishmania donovani causing cutaneous leishmaniasis in Sri Lanka: a wolf in sheep's clothing? Trends in Parasitology 2009; 25: 458 - 463.

10. Weekly Epidemiological Report Sri Lanka 2011; 38 (1) 3.

11. Weekly Epidemiological Report Sri Lanka 2012; 39 (1) 3.

12. Weekly Epidemiological Report Sri Lanka 2013; 40 (1) 3.

13. Sandanayaka R, Kahawita I, Gamage A, Siribaddana S, Agampodi S. Emergence of cutaneous leishmaniasis in Polonnaruwa, Sri Lanka 2008 - 2011. Tropical Medicine \& International Health 2014; 19 (2) 140 - 145.

14. Ministry of Health of Sri Lanka. Anti-Filariasis Campaign.

http://www.filariasiscampaign.health.gov.lk/subpgs/01_aboutus.html\#3 (accessed on 5 May 2014).

15. Pathmeswaran A, Jayatissa R, Samarasinghe S, Fernando A, de Silva RP, Thattil RO, de Silva NR. Health status of primary schoolchildren in Sri Lanka. Ceylon Medical Journal 2005; 50 (2) 46 - 50. 
16. Gunawardena N, Kumarendran B, Ebenezer R, Gunasingha MS, Pathmeswaran A, de Silva NR. Soil-transmitted helminth infections among plantation-sector schoolchildren in Sri Lanka: prevalence after ten years of preventive chemotherapy. PLoS Neglected Tropical Diseases 2011; 5 (9) e1591.

17. Gunawardena S, Gunawardena NK, Kahathuduwa G, Karunaweera ND, de Silva NR, Ranasinghe UB, Samarasekara SD, Nagodavithana KC, Rao RU, Rebollo MP, Weil GJ. Integrated school-based surveillance for soil-transmitted helminth infections and lymphatic filariasis in Gampaha District, Sri Lanka. American Journal of Tropical Medicine \& Hygiene 2014; 90 (4): 661-666.

18. Soil-transmitted helminthiases: eliminating soil-transmitted helminthiases as a public health problem in children. Progress report 2001-2010 and strategic plan 2011-2020. Geneva, World Health Organization, 2012. (WHO/HTM/NTD/PCT/2012.4)

19. Ministry of Health of Sri Lanka. Guidelines on de-worming children and pregnant women in community setting 2013 - 2016. General Circular Letter No 02-172 / 2012. Accessed at http://www.familyhealth.gov.lk/web/index.php?option=com_phocadownload\&view=category\&id=59\&Itemid=14 6\&lang=en on 14 May 2014.

20. De Silva NR \& Jayawickrama H. Can we eliminate soil-transmitted helminth infections in Sri Lanka? Ceylon Medical Journal 2012; 57 (1): 1-4.

21. Smith J \& Taylor EM. MDGs and NTDs: re-shaping the global health agenda. PLoS Neglected Tropical Diseases 2013; 7 (12): e2529.

22. Editorial. Neglected Tropical Diseases: becoming less neglected. Lancet 2014; 383: 1269.

\section{Submit your next Manuscript to Anuradhapura Medical Journal}

Submit your manuscript at WWw.sljol.info/index.php/AMJ/ 